\title{
Multiple Myeloma: Clinico-hematological Profile in A Tertiary Care Hospital: A Three Years Study
}

\author{
Rajni Kaushik, Rajneesh K. Thakur*, Anchana Gulati and Sudarshan K. Sharma \\ Department of Pathology, Indira Gandhi Medical College, Shimla. India
}

\section{ABSTRACT}

Background: To analyze the clinical findings and haematological profile of multiple myeloma patients.

Material and methods: All newly diagnosed patients of multiple myeloma between January' 2013 to December’ 2015 in the department of pathology, Indira Gandhi Medical College, Shimla were the study subjects. The history and clinical findings were recorded. All relevant blood, radiological investigations including peripheral smears, bone marrow aspiration and biopsy were done.

Results: Fifty one patients were diagnosed with multiple myeloma and they comprised $19 \%$ of all haematological malignancies. Out of these, 30 were males and 21 females with a mean age of presentation 58.38 years. The commonest presenting complaint was bone pain followed by fever, CKD and bleeding disorder. Common clinical findings were anemia, osteolytic lesions and renal insufficiency. M band was seen in $83 \%$ of patients on serum protein electrophoresis. On bone marrow examination, majority (53\%) of patients had plasmablastic morphology while, $47 \%$ had plasmacytic features. Diffuse pattern of infiltration on bone marrow biopsy was observed most commonly in $68.3 \%$ patients.

Conclusion: The present study concluded that multiple myeloma is a disease of middle age and elderly with male preponderance. The clinical presentation varies with bone pain being the most common presenting complaint. Morphologically,plasmablastic morphology predominated on bone marrow aspiration while diffuse pattern of infiltration was observed on bone marrow biopsy.

Keywords: Multiple Myeloma, Bone Marrow.

\section{Introduction}

"Multiple myeloma" a term coined by Von Rustizky in 1873 , is a bone marrow based clonal expansion of B lineage plasma cells associated with production of monoclonal immunoglobulins (Ig) or Ig fragments in serum and/or urine. ${ }^{[1]}$ It is included in the category of mature B-cell neoplasms in WHO classification of hematopoietic and lymphoid neoplasms..$^{[2,3]}$

It accounts for $1 \%$ of all neoplastic disorders and $10-15 \%$ of hematological malignancies. Multiple myeloma is seen in all races, with a higher incidence in African Americans in comparison to Asian. Multiple myeloma (MM) is a disease predominantly of middle aged and elderly. ${ }^{[1]}$ The median age of presentation in India is a decade lesser around 55-62 years ${ }^{[4-7]}$ in comparison to Western countries.

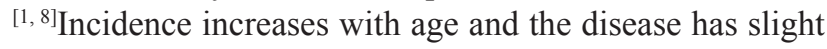
male predilection. ${ }^{[1]}$

Though the etiology of the disease is largely unknown various factors like environmental, occupational, radiation exposure, metal industries, benzene exposure and preexisting medical conditions have been found to increase the incidence of MM. ${ }^{[1]}$ The biological behaviour of myeloma cells depends upon complex and dynamic interplay between myeloma cells and BM microenvironment. This interaction is mediated by a variety of adhesion molecules, cytokines and receptors. The IL-6 is the most important proliferation and survival factor in myeloma. ${ }^{[9]}$

The clinical manifestations arise as a result of bone marrow infiltration by clonal plasma cells and secretion of $\mathrm{M}$ proteins. These are characterized by a clinical pentad: anemia, M protein in the serum/ urine or both, abnormal bone radiographs and bone pain, hypercalcemia, and renal insufficiency/ failure. ${ }^{[1]}$ Bone pain is the commonest presenting symptom with most of the patients having punched out lytic lesions, osteoporosis, osteopenia or fractures on radiological examination. ${ }^{[1,8]}$

Bleeding manifestations are a result of adsorption of clotting factors by $\mathrm{M}$ protein, decrease in clotting factors II, V, VII, VIII, X, and fibrinogen, hyperviscosity and thrombocytopenia. Thrombocytopenia is uncommon in early phases of myeloma, possibly because of the thrombopoietic activity of IL-6. However, thrombocytopenia may develop subsequent to therapy or from autoimmune mechanisms. ${ }^{[8]}$ There is increased tendency to acquire infections due to immune deficiency resulting from diffuse hypogammaglobulinemia. ${ }^{[8]}$ Renal involvement is most common in patients having Bence Jones (free light chain only) myeloma and IgD type of myeloma. ${ }^{[10]}$ 
Majority of patients show normocytic normochromic anemia and rouleaux formation. Sometimes, leukoerythroblastic blood picture or plasma cell leukemia is also seen. ${ }^{[1]}$ Bone marrow examination along with clinical, laboratory and radiological parametersis essential for establishing the diagnosis of Multiple myeloma. Plasma cell morphology, percentage and pattern of infiltration of plasma cells in marrow have significant correlation with the clinical stage and survival. Higher percentage of Plasma cell fraction is a reliable predictor of relapse in treated MM patients and it also helps to evaluate morphological remission and minimal residual disease in MM patients. ${ }^{[12,13]}$

The present study was undertakenwith the aim to analyze the clinico-hematological profile of multiple myeloma patients in IGMC Shimla, a tertiary care hospital of Himachal Pradesh.

\section{Material and Methods}

The study included all newly diagnosed patients of multiple myeloma from Jan 2013 to Dec 2015. The patients on treatment and follow up were excluded. Patients were diagnosed as per the WHO criteria. Relevant history, clinical and radiological findings of the patients were recorded. Complete hemogram, peripheral smears, biochemical investigations, serum protein electrophoresis and urine protein electrophoresis were done. A written, informed consent was taken from the patients. Bone marrow aspiration and biopsy were performed in all the cases suspected of having multiple myeloma.

\section{Results}

Out of total 952 bone marrow examinations performed from Jan 2013 to Dec 2015, 264 cases had hematological malignancies. Out of these 51 were multiple myeloma cases which accounted for $19 \%$ of hematological malignancies. The age of patients ranged from 23 to 90 years with a mean of 58.38 years. The male: female ratio was $1.4: 1$.

The commonest presenting complaintwas bone pain comprising $62.74 \%$ of patients followed by generalized weakness $(23.52 \%)$, fever $(15.68 \%)$, renal involvement $(15.68 \%)$ and bleeding disorders in $(8 \%)$ patients. Majority
(94\%) of the patients had anemia, followed by bony tenderness (47\%), splenomegaly (9.8\%), hepatomegaly (7.8\%) and lymphadenopathy (6\%). Bone lesions included lytic lesions $(53 \%)$, osteoporosis $(19.6 \%)$, pathological fractures (15.6\%)and osteosclerotic lesions (4\%).

The results of lab investigations revealed that, majority of the patients had moderate $(8-10 \mathrm{~g} / \mathrm{dl})$ to severe anemia $(<8 \mathrm{~g} / \mathrm{dl})$ in $45 \%$ each and mild anemia in $4 \%$ patients. Six percent patients did not have anemia. On peripheral smear findings normocytic, normochromic anemia was observed in majority i.e. $70.59 \%$ patients followed by macrocytic in $23.53 \%$ and microcytic in $5.85 \%$. Short rouleaux formation was seen in $70 \%$ patients. Twelve percent patients presented with leukoerythroblastic blood picture. ESR was raised in all the patients however, ESR (>100 $\mathrm{mm}$ in $1^{\text {st }}$ hour) was observed in $51 \%$ patients. Majority i.e. $72 \%$ patients had total leukocyte count WNL. However leucopenia was seen in $22 \%$ and leucocytosis in $6 \%$ patients. Thrombocytopenia was seen in $23.53 \%$ of patients, rest of them had normal platelet count. Serum Calcium levels $>11 \mathrm{mg} / \mathrm{dl}$ (hypercalcemia) were observed in $11.76 \%$ patients. On Serum protein electrophoresis (SPEP), M band was detected in $83 \%$ patients whereas $17 \%$ patients did not have $\mathrm{M}$ band.

Plasma cell morphology on bone marrow aspiration was predominantly plasmablastic (poorly differentiated type of myeloma cells) in 53\% followed by plasmacytic (well differentiated and intermediately differentiated) in $47 \%$ cases (Figure 1, 2).

Majority of the patients i.e. $82.35 \%$ had stage III ( $>50 \%$ of plasma cells on bone marrow biopsy) disease at the time of presentation (Table 1). Patterns of infiltration on BM biopsy in decreasing order of frequency were diffuse in $68.62 \%$ patients followed by mixed $21.57 \%$, interstitial $7.85 \%$ and paratrabecular in $1.96 \%$ patients (Table 2, Figure 3-5).

\section{Discussion}

Multiple myeloma comprises $10-15 \%$ ofall hematological malignancies. ${ }^{[1]}$ In the present study we observed multiple myeloma patients accounting for $19 \%$ of all the

Table 1: Percentage of plasma cells on BM biopsy \& staging

\begin{tabular}{|c|c|c|c|}
\hline \% of plasma cells & Stage & Number of patients $(\mathbf{n = 5 1 )}$ & (\%) \\
\hline$<20$ & I & 2 & 3.92 \\
\hline $20-50$ & II & 7 & 13.73 \\
\hline$>50$ & III & 42 & 82.35 \\
\hline
\end{tabular}


Table 2: Pattern of infiltration of bone marrow

\begin{tabular}{|l|l|l|}
\hline Pattern of infiltration & Number of patients(n=51) & $(\%)$ \\
\hline Interstitial & 04 & 7.85 \\
\hline Nodular & - & - \\
\hline Mixed & 11 & 21.57 \\
\hline Diffuse & 35 & 68.62 \\
\hline Paratrabecular & 01 & 1.96 \\
\hline
\end{tabular}

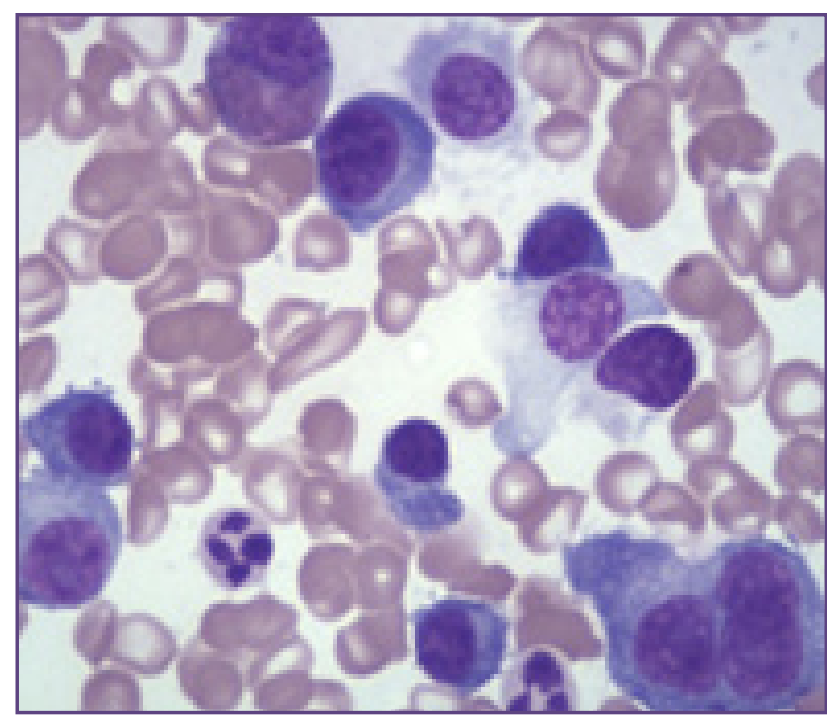

Fig. 1: Bone Marrow aspiration(Giemsa stain, 100X): Mature and immatureneoplastic plasma (myeloma) cells.

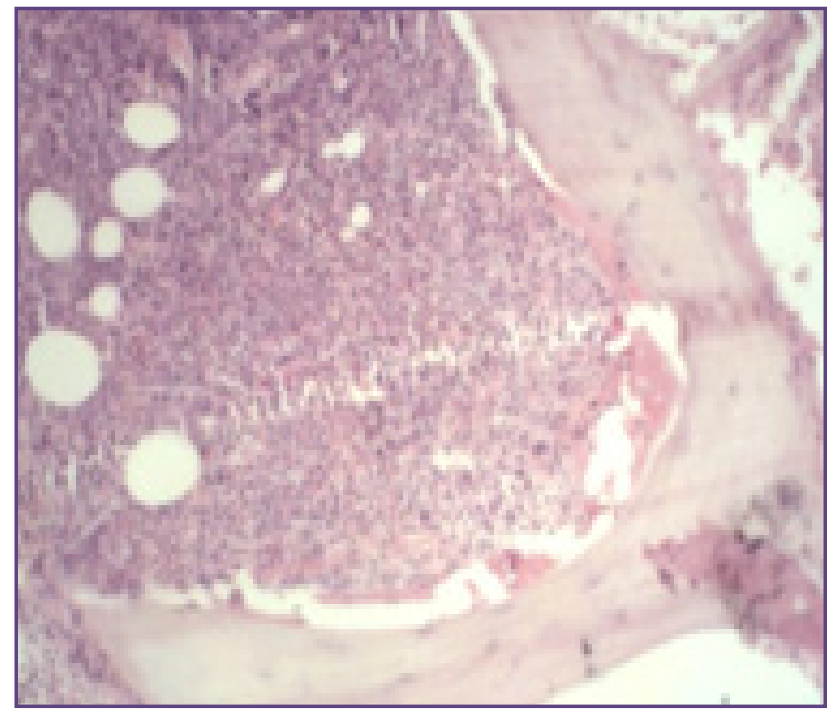

Fig. 3: BM trephine biopsy sections (Paraffin embedded,H \&E stain, 10X) showing Diffuse infiltration (packedmarrow).

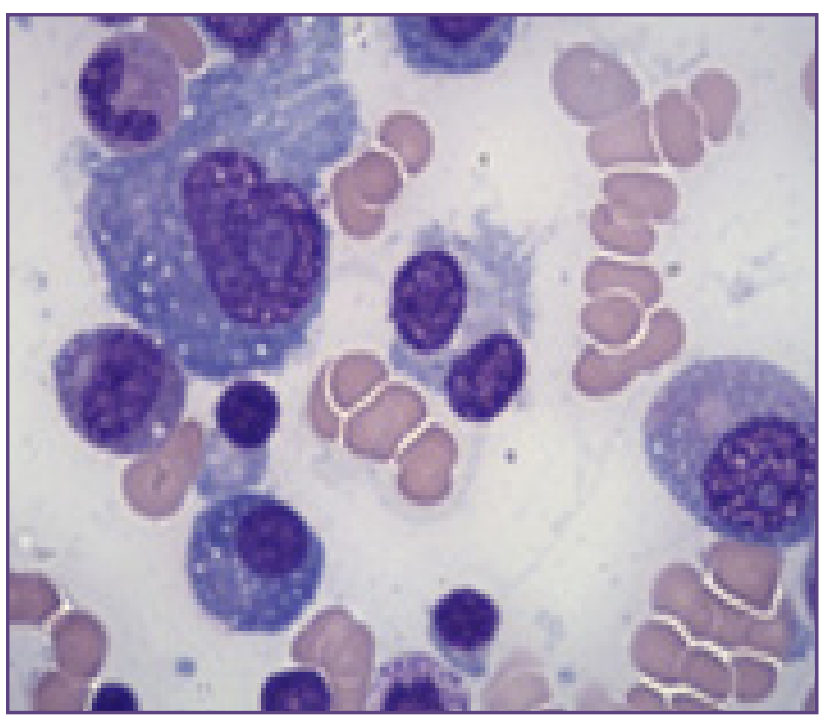

Fig. 2: Bone Marrow aspiration (Giemsa stain, 100X): Plasmablastic plasma cell morphology.

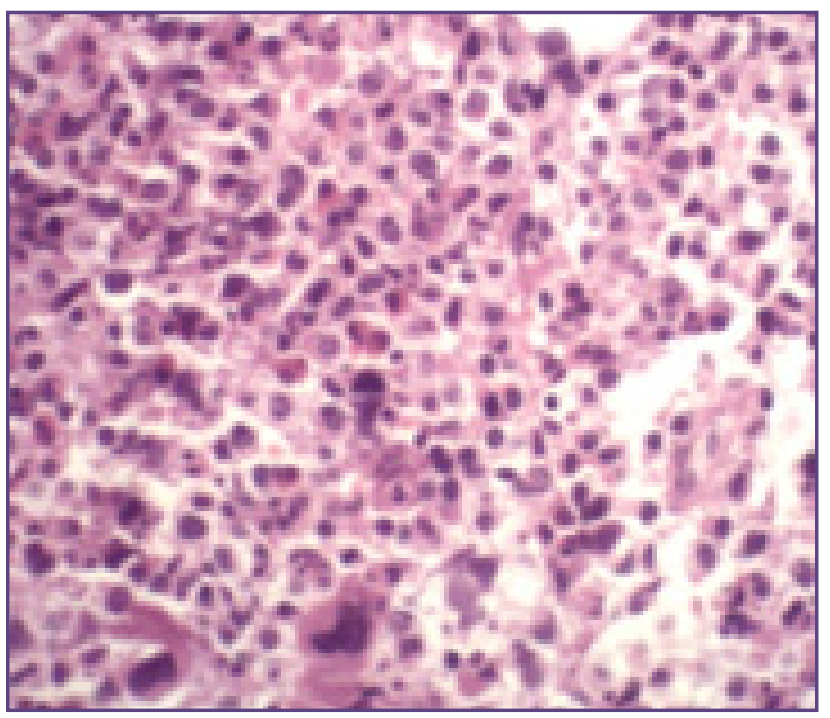

Fig. 3: BM trephine biopsy sections (Paraffin embedded,H \&E stain, 10X) showing Diffuse infiltration (packedmarrow). 


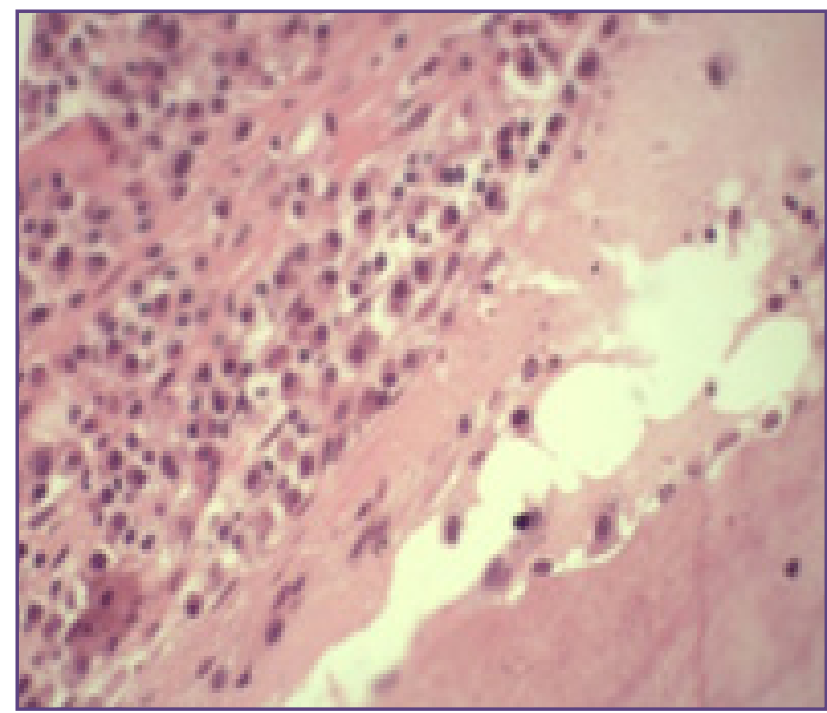

Fig. 5: BM trephine biopsy sections (Paraffin embedded,H \&E stain, 40X) showing Paratrabecular pattern of infiltration.

hematological malignancieswhich is higher in comparison to reports by Diwan G et al $(13 \%),{ }^{[14]}$ Kaur et al $(11 \%)^{[5]}$ and Rajkumar S.V. (10\%). ${ }^{[15]}$ The higher percentage in our study may be due to our institute being the only Regional cancer treatment center and tertiary care referral hospital in our state.

The disease predominantly affects middle aged and elderly and is unusual in young patients with approximately $2 \%$ beingless than 40 years and still rarer in patients younger than 30years. ${ }^{[16]}$ We also observed only one patient less than 40 years. The mean age of presentation was 58.38 years which is in accordance with other studies ${ }^{[4-7,15]}$ whereas it is lower than that observed by Diwan G et al (62 years $)^{[14]}$ and Rajkumar S.V. (65 years). ${ }^{[15]}$

Present study revealed male preponderance ( M:F- 1.4:1) as seen in other studies ranging from $1.5: 1$ to $2: 1^{[4,5,7]}$ whereas both sexes were equally involved in a study by Diwan et al. ${ }^{[14]}$

These patients have variable clinical presentation. We observed bone painas chief presenting complaint in $63 \%$ patients. These are higher than observed by Kyle et al and Kaur et al. 58\% and 50\% respectively ${ }^{[5,18]}$ however lower than observed by Diwan et al (85\%) and Subramanian $\mathrm{R}$ et al. $(82 \%) \cdot{ }^{[4,14]}$ Bone pain was followed by generalized weakness $(23.52 \%)$ which was lower than Diwan et al $(55 \%)$ and Kyle et al (32\%). ${ }^{[4,18]}$ Sixteen percent patients came with fever which was higher than Kyle et al (0.7\%) whereas lower than that observed by Diwan et al (35\%). Renal involvement in our study (16\%) was quite lower

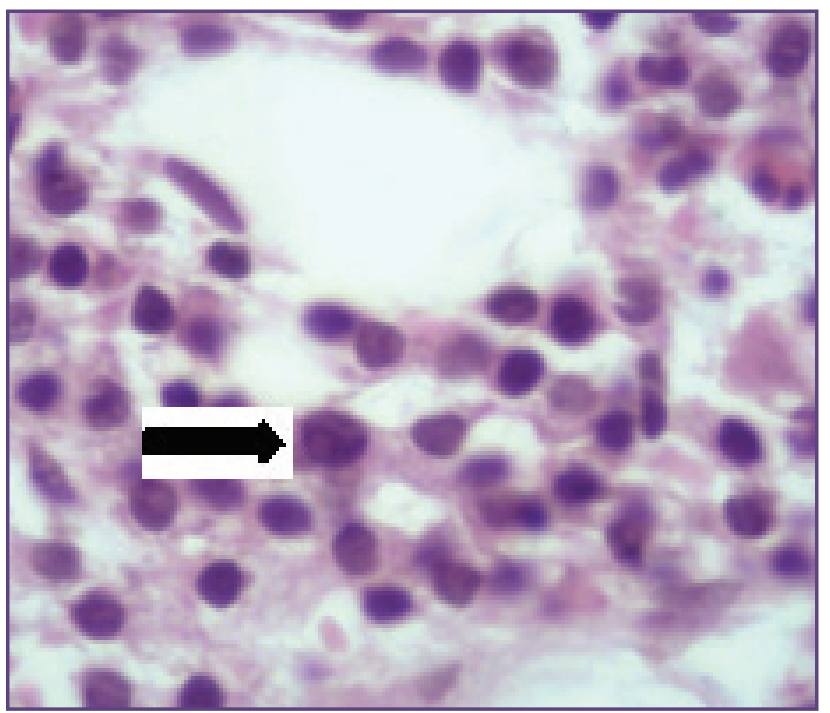

Fig. 6: BM trephine biopsy sections (Paraffin - embedded,H \&E stain, 100X) Dutcher body shown by $(\rightarrow)$

than Subramanian R et al. (36\%) and Diwan et al. (30\%). ${ }^{[4,14]}$ Bleeding manifestations in present study (8\%) were comparable to Diwan et al $(10 \%) .^{[14]}$

We found splenomegaly in $9.8 \%$, hepatomegaly in $7.8 \%$ and lymphadenopathy in $6 \%$ of myeloma patients. It was quite higher in comparison to study by Kyle et al who observed splenomegaly (1\%), hepatomegaly (4\%) and lymphadenopathy in $(1 \%)$ of patients however Diwan et al did not encounter organomegaly. ${ }^{[14,18]}$

Lytic lesions which are the hallmark of myeloma patients, were seen in 53\% patients under study and these are well in accordance with other studies in literature. ${ }^{[6,18]}$ Osteoporosis/osteopenia (20\%) the next frequentobservation in thisstudy was comparable to study by Kyle et al $(20 \%)$ while Kalita et al had reported a very high incidence $(52 \%)$ of osteoporosis. ${ }^{[6,18]}$ We found myeloma patients presenting with pathological fracturesin $16 \%$ cases well in tune with literature report of $6-20 \% .^{[6,18]}$ However none of the studies have reported osteosclerotic lesions which we found in $4 \%$ of our patients and have been mentioned in literature also. ${ }^{[1,8]}$

Multiple myeloma patients have increased ESR due to anemia and increased paraproteins. ESR $>100 \mathrm{~mm}$ in $1^{\text {st }}$ hour is reported in different series from $33-100 \% .^{[4,5,14,18]}$ Our observation (51\% patients) is similar to these studies.

Anemia in myelomais caused due to replacement of marrow by myeloma cells and decreased production of erythropoietin due to accompanying renal involvement. In some cases it may be associated with cytokine mediated 
BM suppression. ${ }^{[1]}$ Severe anemia $(\mathrm{Hb} \text { level }<8 \mathrm{~g} / \mathrm{dl})^{[19]}$ was seen in $45 \%$ patients in the present study. Though lower number of patients having severe anemia were observed as compared to Kaur et al (75\%) and Subramanian R et al (71\%) but higher than Kalita L K et al (33\%) and Diwan et al $(25 \%)$. $^{[-6,14]}$

In most patients there is increased rouleaux formation and increased background basophilic staining due to the presence of paraproteinin the blood. ${ }^{[8]}$ Rouleaux formation was seen in $70 \%$ of our patients which is lower in comparison to observations by Subramanian $\mathrm{R}$ et al (91\%)and Kaur et al (82\%), though higher than Diwan et al $(35 \%))^{[4,5,14]}$

Thrombocytopenia is uncommon in early phases of myeloma, possibly because of thrombopoietic activity of IL-6. However, thrombocytopenia may develop subsequently due to therapy or from autoimmune mechanisms. ${ }^{[8]}$ Thrombocytopenia in our patients $(24 \%)$ was comparable to observations made by Kaur et al (25\%) [5], however higher than Diwan et al and Kyle et al $10 \%$ and $5 \%$ respectively. ${ }^{[14,18]}$

M band is detectable in $97 \%$ of myeloma patients they may have either intact immunoglobulin or fragment or a free light chain on serum / urine protein electrophoresis whereas cases with non-detectable monoclonal proteins are referred to as non-secretors (1-3\%) [1]. M band was detectable in $83 \%$ of our patients which is slightly higher than that observed by Kaur et al (75.4\%)while lower than Diwan et al who found it in all the patients and Kyle et al in $(93 \%)$ patients. ${ }^{[5,14,18]}$

The bone marrow aspiration revealstwo types of plasma cell morphology (plasmacytic and plasmablastic), the plasmablastic morphology correlates well with poor prognosis. ${ }^{[12]} \mathrm{We}$ found predominantly plasmablastic morphology in majority (53\%) where as plasmacytic morphology was observed in (47\%) myeloma patients. The observation was in concordance with study by Kaur et al [plasmacytic in (39\%) and plasmablastic in (61\%)] and Subramanian $\mathrm{R}$ et al [plasmacytic in (44\%) and plasmablastic in $(56 \%)$ ] who also found plasmablastic morphology predominantly. ${ }^{[4,5]}$

Another indicator of high prognostic significance is percent infiltration of myeloma cells in bone marrow biopsy (more than $50 \%$ plasma cell infiltration stage III). ${ }^{[12]}$ Most of our patients $(82 \%)$ were in stage III, which is higher than that observed by Subramanian R et al (71\%),Kaur et al (64.3\%) and Kalita LK et al (26\%). ${ }^{[4-6]}$

Various patterns of infiltration of marrow like interstitial, focal/nodular, diffuse, mixed and paratrabecular are observed in myeloma patients.A packed/diffuse marrow indicates worse prognosis. ${ }^{[10]}$ In our study majority of cases had diffuse involvement $(68.62 \%)$ followed by mixed (21.57\%), interstitial (7.85\%) and paratrabecular (1.96\%). Similarly diffuse infiltration was also the predominant pattern of infiltration in other studies. ${ }^{[4,5]}$

\section{Conclusion}

The present study concluded that multiple myeloma is a disease of middle aged and elderly with male preponderance. They accounted for slightly higher percentage of haematological malignancies in present study in comparison to literature. This may be due to our institute being the only Regional cancer treatment center and tertiary care referral hospital in our state. Bone pain was the most common presenting complaint along with lytic lesions on skeletal survey. Osteosclerotic lesions were also observed. Majority of our patients had plasmablastic morphology on bone marrow aspiration while bone marrow biopsy revealed majority of patients in stage III with predominantly diffuse pattern of infiltration.Hence most of our patients presented in advanced clinical stage.

\section{References}

1. Dispenzieri A, Lacy MQ, Griepp PR. Multiple Myeloma. In: Greer JP, Foerster J, Rodgers GM, Paraskevas F, Glader B, Arber DA, editors. Wintrobe's Clinical Hematology 12th ed. Philadelphia: Lippincott Williams \& Wilkins; 2009:2373-2438.

2. Jaffe ES, Nancy LH, Harald S, Peter GI. Classification of lymphoid neoplasms: the microscope as a tool for disease discovery. Blood Jr. 2008 ; 112(12): 4384-4399.

3. Swerdlow SH, Campo E, Pileri SA, Harris NL, Stein H, Siebert R et al. The 2016 revision of the World Health Organization classification of lymphoid neoplasms. Blood Jr. 2016; 127(20): 2375-2390.

4. Subramanian R, Basu D, Dutta TK. Prognostic significance of bone marrow histology in multiple myeloma. Indian $\mathrm{J}$. Cancer. 2009; 46(1): 40-5.

5. Kaur P, Shah BS, Bajaj P. Multiple myeloma: A clinical and pathological profile. G. J.O. 2014; 16: 14-20.

6. Kalita LK, Kalita C, Gogoi PK et al. A clinic- epidemiological study of multiple myeloma- A hospital based study at Gauhati Medical College and Hospital, Guwahati, Assam. J. Evid. Based Med. Health. 2016; 3(36), 1788- 1794.

7. National cancer registry programme. Consolidated report of population based cancer registries 1990- 1996, Indian Council of medical research, New Delhi. 2001.

8. Lichtman MA, Kipps TJ, Seligsohn U, Kaushansky K, Prchal JT. Williams Hematology. 8thed. New York, NY : McGraw Professional; 2010.

9. Kuehl WM, Bergsagel PL. Molecular pathogenesis of multiple myeloma and its premalignant precursor. J Clin Invest. 2012; 122(10): 3456- 3463. 
10. Heher EC, Rennke HG, Laubach JP, Richardson PG. Kidney Disease and Multiple Myeloma. Clin J Am SocNephrol 8: 2007-2017, 2013. doi: 10.2215/CJN.12231212

11. Bain BJ, Clark DM, Wilkins BS. The normal bone marrow. In: Bain BJ, Clark DM, Wilkins BS, editors. Bone Marrow Pathology. 4th ed. Singapore: WileyBlackwell; 2010:421-60.

12. Bartl R, Frisch B, Burkhardt R, Fateh-Moghadam A, Mahl $\mathrm{G}$, Gierster $\mathrm{P}$ et al . Bone marrow histology in myeloma: Its importance in diagnosis, prognosis, classification and staging. Br J Haematol 1982; 51:361-75.

13. Stifter S, Babarovic E, Valkovic T, Bekafigo IS, Stemberger $\mathrm{C}$, Nacinovic A et al. Combined evaluation of bone marrow aspirate and biopsy is superior in the prognosis of multiple myeloma. Diagnostic Pathology 2010; 5: 30.

14. Diwan AG, Gandhi SA, Krishna K, Shinde VP. Clinical profile of the spectrum of multiple myeloma in a teaching hospital. Medical Jr. of Dr. D. Y. Patil University. 2014; 7(2): 185- 8
15. Rajkumar SV, Greipp PR. Prognostic factors in multiple myeloma. HaematolOncolClin North Am 1999; 13:1295.

16. Kapoor R, Bansal M, Sastri GJ, Sandhu MS, Garg M, Sharma SC.Clinical Spectrum and Prognosis of MultipleMyeloma in Patients Younger than 30 Years : Is it Different from the Elderly ?2006;8 ( 4): 225-228.

17. Guadarrama MBR, Medina CAM, Martinez EA. Plasma Cell Neoplasms, Clinicopathological Characteristics and Immunophenotype of 21 Patients. OJPathology. 2012; $2: 127-132$.

18. Kyle RA, Gertz MA, Witzig TE, Lust JA, Lacy MQ, Dispenzieri A et al. Review of 1027 Patients With Newly Diagnosed Multiple Myeloma. Mayo Clin Proc. 2003; 78: 21-23.

19. Haemoglobin concentrations for the diagnosis of anaemia and assessment of severity. Vitamin and Mineral Nutrition Information System. Geneva, World Health Organization, $2011(\mathrm{WHO} / \mathrm{NMH} / \mathrm{NHD} / \mathrm{MNM} / 11.1)$

*Corresponding author:

Dr. Rajneesh K. Thakur, Junior Resident Department of Pathology, Indira Gandhi Medical College, Shimla. (India),

Phone: +91 9418067508, 9736579407

Email: rajneesh.thakur11@gmail.com,

Date of Submission : 02.01.2017

Date of Acceptance : 20.07.2017

Financial or other Competing Interests: None.

Date of Publication : 25.10.2017 